Martínez Clares, Pilar; Martínez Juárez, Mirian; Pérez Cusó, Javier (2014). Tutoría Universitaria: entorno emergente en la Universidad Europea. Un estudio en la Facultad de Educación de la Universidad de Murcia. Revista de Investigación Educativa, 32 (1),111-138.

http://dx.doi.org/10.6018/rie.32.1.148411

\title{
TUTORÍA UNIVERSITARIA: ENTORNO EMERGENTE EN LA UNIVERSIDAD EUROPEA. UN ESTUDIO EN LA FACULTAD DE EDUCACIÓN DE LA UNIVERSIDAD DE MURCIA
}

\author{
Pilar Martínez Clares, Mirian Martínez Juárez y Javier Pérez Cusó \\ Departamento de Métodos de Investigación y Diagnóstico en Educación \\ Facultad de Educación. Universidad de Murcia
}

\section{RESUMEN}

La actual realidad de la Educación Superior comporta una serie de cambios que permiten intuir el nuevo papel que debe desempeñar la tutoría universitaria. Cuando se percibe como factor de calidad e innovación, demanda nuevas responsabilidades así como renovados modos de hacer y actuar. Ante el protagonismo que adquiere la tutoría en la cultura universitaria europea de los centros, nos cuestionamos si, efectivamente, los cambios que requiere se están llevando a la práctica en nuestro contexto y cómo son percibidos por los verdaderos actores del proceso de aprendizaje, el alumnado. El estudio realizado en la Facultad de Educación de la Universidad de Murcia permite concluir que, si bien existe sensibilidad ante la misma, falta una mayor difusión, divulgación, compromiso e integración de la tutoría desde la propia institución, pues no está reconocida y los estudiantes la conciben con un carácter excesivamente académico.

Palabras clave: Tutoría universitaria; Educación Superior; Calidad, Innovación.

\footnotetext{
Correspondencia:

Departamento de Métodos de Investigación y Diagnóstico en Educación

Facultad de Educación. Universidad de Murcia

Pilar Martínez Clares. E-mail: pmclares@um.es

Mirian Martínez Juárez E-mail: mmartinez@um.es

Javier Pérez Cusó. E-mail: javierperezcuso@um.es
} 


\title{
UNIVERSITY TUTORIALS AND SUPPORT SESSIONS:AN EMERGING ENVIRONMENT AT EUROPEAN UNIVERSITIES.A STUDY ATTHE FACULTY OF EDUCATION AT THE UNIVERSITY OF MURCIA
}

\begin{abstract}
Higher Education nowadays involves changes which reveal the new role of university tutorials and student support and guidance services. Tutorials and support sessions are perceived as quality and innovation factors, which require new responsibilities and know-how. Considering the central role played by tutorials and support sessions in European universities, this paper questions whether the changes required are indeed being implemented in our context, and how they are perceived by key players in the learning process, namely students. This study was conducted at the Faculty of Education (University of Murcia, Spain). The results show that there is a need for further information, integration and commitment to tutorials and support sessions, as they are not adequately appraised, and students perceive them as academic sessions.

Keywords: University Tutoring; Higher Education; Quality; Innovation.
\end{abstract}

\section{INTRODUCCIÓN}

En la actualidad, la tutoría universitaria resulta singularmente apropiada y necesaria para dar respuesta a las demandas del nuevo modelo universitario. El escenario de la transformación inspirado en Bolonia está generando un nuevo espacio o entorno que resulta apropiado para la promoción de la tutoría "como un valioso espacio colaborativo de dinamización personalizada del aprendizaje y de constitución de una comunidad de iniciación, discurso y reflexión" (Lapeña, Sauleda \& Martínez, 2011, p. 343). Hacer realidad este modelo comporta impulsar cambios sustanciales que implican la adaptación de las metodologías de enseñanza y los objetivos formativos, la innovación en las funciones y técnicas docentes, la institucionalización de la tutoría y la atención personalizada (Sanz Díaz, 2012).

Para la Educación Superior uno de los mayores avances que aporta el Proceso de Bolonia es el establecimiento y consolidación de los sistemas de garantía de calidad. Estos sistemas de calidad constituyen un marco de reconocimiento y confianza mutua tanto para las instituciones de Educación Superior como para los diferentes agentes implicados, conforme a los criterios, procedimientos y directrices establecidos a nivel europeo; es por ello que la universidad de hoy, del siglo XXI, considera la tutoría como un factor o elemento de calidad, de innovación y de futuro, en definitiva, como un valor al alza (Martínez Clares, Martínez Juárez \& Pérez Cusó, 2012).

La integración de esta temática no es algo nuevo en esta etapa educativa tal y como se contempla tanto en los Estatutos como en los Reglamentos de las Universidades Españolas. No obstante, comienza a suscitar mucho interés, sobre todo desde hace aproximadamente una década, beneficio que se refleja en numerosos trabajos y estudios, entre los que destacamos a Gallego (1997), Almajano y Bertrán (1999), Monreal (1999), Sebastián y Sánchez (1999), Alañón (2000), Salmerón y López (2000), Villayandre y Pérez (2000), Cáceres y Rodríguez Monzonís (2001), Filella et al. (2001), Álvarez Pérez (2002), Lázaro (2003), Álvarez y Jiménez (2003), Ferrer (2003), Lobato (2003), Zabalza (2003), Domínguez (2004), García Nieto, Asensio, Carballo, García García y Guardia 
(2004), Coriat y Sanz (2005), Álvarez y González (2008), Rodríguez Espinar (2008), Cano (2009) o Sanz Oro (2009).

Estos trabajos ponen en evidencia la importancia que tiene la tutoría, no sólo en la labor docente, sino también en la formación integral de nuestros futuros titulados. Todos estos estudios revelan que la tutoría en el marco de la Educación Superior experimenta en los últimos años un gran desarrollo. En nuestros días, todas las Universidades y sus distintas facultades y centros están poniendo en práctica planes de acción tutorial para favorecer y potenciar el desarrollo integral de sus estudiantes como parte de la formación a lo largo de la vida.

En esta línea, Sanz Oro (2005) declara que todos los cambios producidos por la convergencia europea intuyen el nuevo papel que debe pasar a desempeñar la tutoría universitaria, que tendrá que adaptarse a las nuevas características y necesidades de la institución y del alumnado, por lo que tendrá que implicarse en cuestiones tales como: a) conocer al alumnado, b) crear entornos de aprendizaje, c) inculcar valores, d) fomentar el desarrollo comunitario, e) conocer y acceder a los recursos de la institución, f) crear recursos humanos y materiales, g) establecer programas de formación de tutores, h) establecer relaciones de colaboración entre profesores tutores, i) reconsiderar las políticas y las prácticas profesionales y j) estar alerta a los problemas personales que puedan inhibir el aprendizaje.

Esta nueva realidad de la Educación Superior en el EEES supone, entre otros aspectos, una docencia centrada en el estudiante, en su aprendizaje, donde el papel del profesorado es el de guía y mediador de su proceso formativo, una formación a lo largo de la vida, orientada a la consecución de competencias.

Todo ello involucra la presencia de la acción tutorial como factor de calidad e innovación. Este nuevo escenario considera que la tutoría universitaria debe trascender la simple atención a las dudas inmediatas que inquietan a los estudiantes, como expresa Cano (2009, p. 183), "más allá de la típica consulta de escaparate", contemplada, exclusivamente, dentro de las seis horas reglamentarias que dedica cada profesor a la atención de sus alumnos. La tutoría, en este contexto como en todos, se tiene que integrar como un proceso educativo más y, para ello, tiene que tener un respaldo institucional, una normativa que la regule y una integración en la función docente.

Respecto a la normativa universitaria en esta temática, vislumbramos que es muy variada y compleja y encontramos legislación estatal y autonómica, además de la normativa propia de cada universidad. En el plano estatal, destacamos la Ley Orgánica 6/2001, de 21 de diciembre, de Universidades, modificada por la Ley Orgánica 4/2007, de 12 de abril, y el Estatuto del Estudiante (Real Decreto 1791/2010, de 30 de diciembre); a nivel autonómico nos encontramos con la Ley 3/2005, de 25 de abril, de Universidades de la Región de Murcia.

Merece especial atención el Estatuto del Estudiante Universitario, que tiene como objeto el desarrollo de los derechos y deberes del estudiante universitario (art. 1) y dedica su Capítulo V a la tutoría universitaria, en el que se definen los principios generales de ésta y se diferencian tres tipos: tutoría de titulación, tutoría de materia y tutoría para alumnos con discapacidad (art. 20, 21 y 22). En dicho Estatuto se define la orientación al estudiante y la configuración de sistemas tutoriales, desarrollados por el profesorado y el personal especializado de forma que integren acciones de información, orientación y apoyo formativo. 
De siempre, la Educación Superior ha proporcionado a sus estudiantes una formación de máxima calidad, pero, si atendemos a los nuevos planteamientos de Bolonia, además y con especial énfasis en la actual Sociedad de la Información y del Conocimiento, la universidad tiene que garantizar a su alumnado una atención personalizada, comprensiva e integral, a través de la orientación (con los servicios que cuenta para ello) y particularmente de la tutoría, en su modalidad personal, grupal y, hoy más que nunca, con la tutoría virtual, para fundamentar y desempeñar cuatro acciones principalmente (adaptación de Cano, 2008):

- Facilitar la toma de contacto inicial con la Universidad y su progresiva adaptación (antes del ingreso y en el primer año fundamentalmente, con una función informativa principalmente).

- Asesorar en la configuración personal y profesional de su itinerario curricular como ciudadanos activos de una sociedad democrática y de derecho, para favorecer ciudadanos críticos y libres (a través de una función de asesoramiento y orientación, durante su estancia en la universidad).

- Guiar la optimización de su rendimiento académico a lo largo de su estancia formativa en la Universidad (función formativa y de asesoramiento).

- Proveer su acceso al mercado laboral y su inserción profesional (no sólo al finalizar los estudios, sino durante la realización de los mismos, para facilitar el desarrollo del proyecto profesional y de vida con una función de orientación profesional principalmente).

Ante esta redefinición de la tutoría universitaria nos preguntamos si, efectivamente, se han iniciado ya los cambios en este sentido y, lo más importante, si son percibidos por los verdaderos actores, los estudiantes. Por esta razón, desde el Departamento de Métodos de Investigación y Diagnóstico en Educación de la Facultad de Educación de la Universidad de Murcia (UMU), surge la iniciativa de conocer el estado de la cuestión en relación a la tutoría en el centro.

\section{PROPÓSITO}

El propósito de este estudio es conocer la necesidad y la realidad de la tutoría universitaria en la Facultad de Educación de la Universidad de Murcia y contrastar las percepciones del alumnado de nuevo ingreso (Grado) con los alumnos más veteranos (Licenciatura y Diplomatura).

\section{OBJETIVOS}

El propósito anterior podemos concretarlo en los siguientes objetivos:

1. Conocer las experiencias previas del alumnado en relación a la tutoría.

2. Conocer y comparar la información que tiene el alumnado sobre la tutoría universitaria y la necesidad e importancia que le concede.

3. Determinar qué demandan los estudiantes a la tutoría y al tutor universitario.

4. Identificar qué tipo de tutoría utilizan los estudiantes y comparar para qué hacen uso de ella.

5. Conocer y categorizar las acciones de mejora que propone el alumnado para el buen desarrollo de la tutoría universitaria. 


\section{METODOLOGÍA}

\section{Diseño de investigación}

El presente estudio se trata de una investigación descriptiva, ya que se detalla una realidad educativa $\mathrm{y}$, a su vez, se miden de manera independiente los conceptos o variables a las que se refiere (Buendía, Colás \& Hernández, 1998). Se basa en un diseño no experimental y transversal de corte cuantitativo, pues nos servimos de la medición numérica y el análisis estadístico para cumplir con los objetivos establecidos.

Dentro de los métodos descriptivos, optamos por el método de encuesta. Este permite dar respuesta a problemas tanto descriptivos como de relación de variables, a través de la formulación de una serie de cuestiones a un grupo de personas que configuran la muestra de estudio y que, por tanto, tienen la capacidad para responder a dichas preguntas, para concluir con la descripción y el establecimiento de relaciones que darán respuesta al propósito de la investigación.

\section{Participantes}

Los participantes en este trabajo son estudiantes de la Facultad de Educación de la Universidad de Murcia, concretamente pertenecientes a $1^{\underline{o}}$ de Grado en Educación Primaria, alumnos que denominaremos de nuevo ingreso o principiantes, y de $3^{\circ}$ y $5^{\circ}$ de la Licenciatura en Pedagogía y de $3^{\circ}$ de la Diplomatura en Educación Social, a los que denominaremos veteranos.

La elección de estas titulaciones está motivada por diferentes factores. En primer lugar, con la intención de representar la mayoría de las titulaciones ofertadas desde el centro. En segundo lugar, el Grado en Educación Primaria es la titulación con más alumnado de todo el centro, por lo que, para equilibrar el número de alumnos veteranos, se ha escogido más de un curso en la titulación de Pedagogía, atendiendo a alumnos que se encuentran en el ecuador de su carrera, por una parte, y, por otra, finalizando sus estudios, ya que, de alguna manera, han podido experimentar ciertas acciones de orientación y tutoría y cuentan con más experiencia.

Nuestros participantes conforman un total de 607 alumnos que se distribuyen tal y como aparece en la Tabla 1.

TABLA 1

DISTRIBUCIÓN DE LOS PARTICIPANTES POR TITULACIÓN Y CURSO

\begin{tabular}{|l|c|c|c|}
\hline \multicolumn{1}{|c|}{ TITULACIÓN } & CURSO & $\mathbf{N}$ & PORCENTAJE \\
\hline Grado en Educación Primaria & $1^{\circ}$ & 377 & $62.1 \%$ \\
\hline Licenciado en Pedagogía & $3^{\circ}$ & 103 & $16.9 \%$ \\
\hline Licenciado en Pedagogía & $5^{\circ}$ & 72 & $11.8 \%$ \\
\hline Diplomado en Educación Social & $3^{\circ}$ & 55 & $9.2 \%$ \\
\hline \multicolumn{2}{|c}{ TOTAL } & 607 & $100 \%$ \\
\hline
\end{tabular}


Su edad está comprendida entre los 18 y los 51 años. El intervalo más numeroso es el comprendido entre los 18-22 años con un 58.9\%. La edad media de los participantes es de 21.8 años.

En cuanto al sexo de los estudiantes, el 74.9\% son mujeres y el 25.1\% hombres.

Los participantes se dividen en dos grupos: alumnos de nuevo ingreso (pertenecientes a $1^{\mathrm{o}}$ de Grado en Educación Primaria), cuyo plan de estudios presenta la exigencia de la tutoría, así como el desarrollo de diferentes acciones orientadoras, todo ello recogido en un Plan de Acción Tutorial (PAT) implantado en el centro y del que son informados al inicio del curso, y alumnos veteranos (pertenecientes a $3^{\circ}$ y 5o de Pedagogía y 3ํㅜㄹ de Educación Social del plan a extinguir), en cuyas titulaciones no se exige la presencia explícita de la tutoría, ni la implantación de ningún PAT en el centro.

\section{Instrumento}

El instrumento de recogida de información se trata de un cuestionario diseñado ad-hoc, el Cuestionario de Tutoría Universitaria (CTU). Está compuesto por 30 ítems, de los cuales 6 son cuestiones cerradas dicotómicas, 4 de elección múltiple, 16 preguntas semicerradas (en las que es preciso graduar la intensidad de la respuesta en las distintas opciones planteadas a partir de una escala tipo Likert con tres niveles de estimación [Mucho-Regular-Nada], incluyendo una opción abierta que se corresponde con Otras) y 4 cuestiones abiertas.

En el presente trabajo nos centraremos en describir y analizar aquellos ítems directamente relacionados con los objetivos propuestos.

\section{Procedimiento}

El procedimiento seguido a lo largo de este estudio responde a las siguientes etapas: en primer lugar se lleva a cabo el diseño del cuestionario (CTU) por el equipo de investigación de este trabajo, seguido de un proceso de validación de contenido de tipo cualitativo a través de un juicio de expertos.

En segundo lugar, se procede a la recogida de información a través de la administración del CTU a mediados del segundo cuatrimestre del curso 2010/2011, con el fin de que los alumnos de Grado hayan tenido la ocasión de experimentar diferentes momentos y situaciones relacionadas con la tutoría, ya que son estudiantes de primer curso. La cumplimentación del cuestionario se realiza en horario de clase y de forma voluntaria, para ello se cuenta con la colaboración del profesorado que en ese momento imparte docencia a nuestros participantes.

En tercer lugar, se analiza la información a través del paquete estadístico SPSS v. 18.0. y, en último lugar, se interpretan los resultados. En cuanto a la información obtenida a partir de las cuestiones abiertas formuladas, éstas se someten a un análisis de contenido que permite obtener la categorización de las respuestas dadas a dichas preguntas para una mejor comprensión, tal y como veremos en el apartado de resultados. 


\section{RESULTADOS}

En cuanto al primer objetivo planteado, se aprecia en el Gráfico 1 como las experiencias previas de los participantes en relación a la tutoría giran en torno a la opción Resolver problemas puntuales de clase, con un $71.08 \%$ para los alumnos de nuevo ingreso y un $58.41 \%$ para los alumnos veteranos. Es curioso, ya que la mayoría de los participantes proceden de la Ley Orgánica 1/1990, de 3 de octubre, de Ordenación General del Sistema Educativo (LOGSE), que reguló e institucionalizó el modelo de orientación y tutoría presente actualmente en nuestro sistema educativo, por lo que las experiencias de los estudiantes deberían centrarse en aspectos menos concretos y coyunturales de la tutoría, o al menos no en un porcentaje tan elevado.

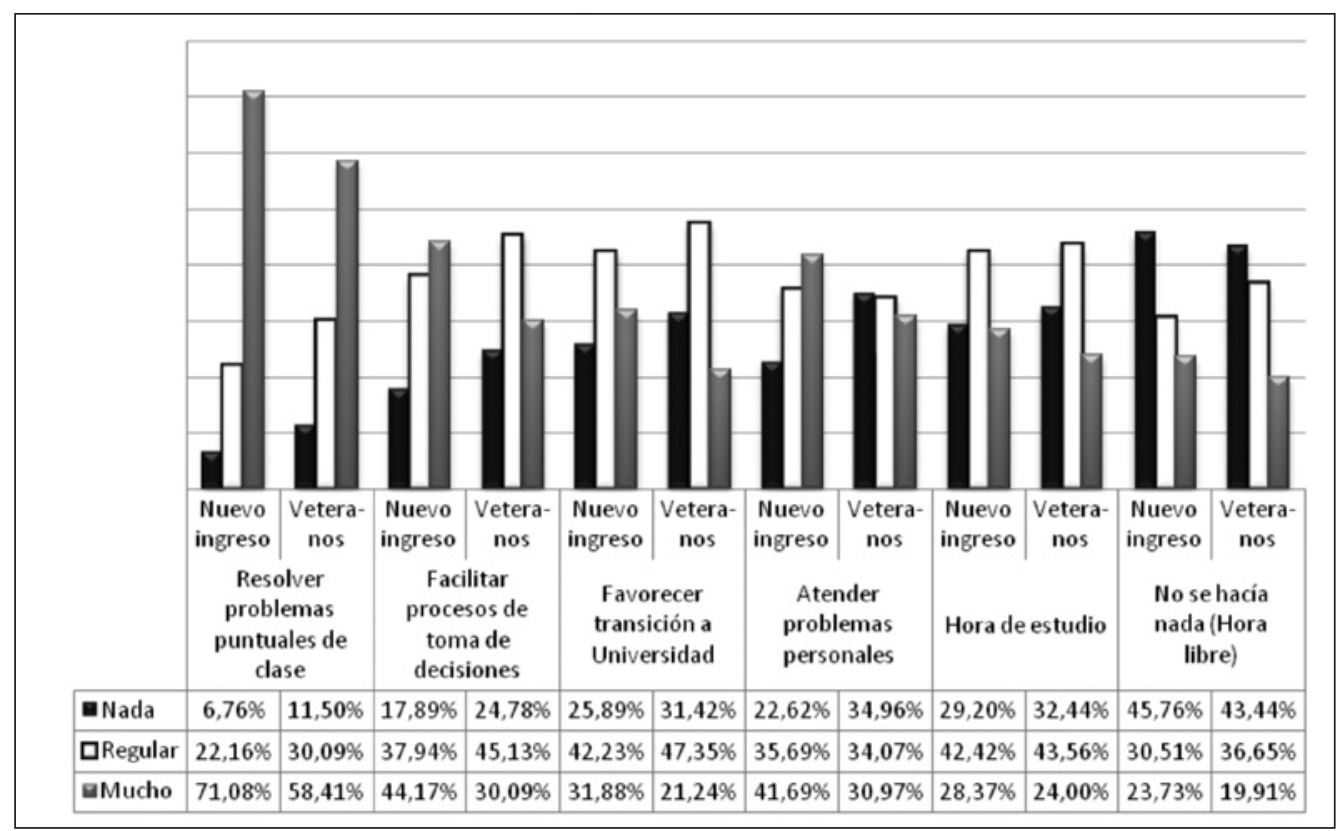

GRÁFICO 1

EXPERIENCIAS PREVIAS DEL ALUMNADO EN RELACIÓN A LA TUTORÍA.

En la Tabla 2 se presentan las diferencias significativas, a partir de la prueba chi cuadrado ( $p<.05)$, en 4 de las opciones planteadas.

En todas ellas, los estudiantes de nuevo ingreso afirman haber vivenciado las alternativas que ofrecen diferencias significativas con mayor frecuencia que los alumnos veteranos. Exceptuando la primera, ya comentada, el resto parecen responder a un concepto de tutoría mucho más actual y relacionado con el proceso continuo, dinámico e integral con el que debe concebirse. Esto nos indica que los estudiantes más nóveles han tenido experiencias mucho más cercanas a la nueva concepción de la tutoría, a pesar de que en etapas no universitarias esta concepción está presente desde el año 1990. Por el contrario, el alumnado veterano destaca por su mayor índice de respuesta 
en el nivel de estimación Nada. Este hecho nos muestra que la práctica de la tutoría en etapas no universitarias continúa evolucionando, pero que aún queda mucho por hacer.

TABLA 2

EXPERIENCIAS PREVIAS DE LOS ALUMNOS EN RELACIÓN A LA TUTORÍA. DIFERENCIAS SIGNIFICATIVAS.

\begin{tabular}{lcccc}
\hline & Chi cuadrado & Gl & Sig \\
\hline $\begin{array}{l}\text { Resolver problemas } \\
\text { puntuales de clase }\end{array}$ & $\begin{array}{c}\text { Nuevo ingreso } \\
\text { Veteranos }\end{array}$ & 10.599 & 2 & .005 \\
\hline $\begin{array}{l}\text { Facilitar procesos de toma } \\
\text { de decisiones }\end{array}$ & $\begin{array}{c}\text { Nuevo ingreso } \\
\text { Veteranos }\end{array}$ & 12.192 & 2 & .002 \\
\hline $\begin{array}{l}\text { Favorecer transición a } \\
\text { Universidad }\end{array}$ & $\begin{array}{c}\text { Nuevo ingreso } \\
\text { Veteranos }\end{array}$ & 8.047 & 2 & 0.18 \\
\hline $\begin{array}{l}\text { Atender problemas } \\
\text { personales }\end{array}$ & $\begin{array}{c}\text { Nuevo ingreso } \\
\text { Veteranos }\end{array}$ & 12.172 & 2 & .002 \\
\hline
\end{tabular}

Para contrastar el segundo de los objetivos (Conocer y comparar la información que tiene el alumnado sobre la tutoría universitaria y la necesidad e importancia que le concede), se pregunta a los participantes si consideran necesaria la tutoría para su formación universitaria. Como se observa en el Gráfico 2, casi el 91\% de los estudiantes responden afirmativamente. Igualmente, también interesa conocer si disponen de información general sobre tutoría (qué es, a quién se dirige, de qué o quiénes depende) a lo que el $53.3 \%$ responde que sí, frente a un $45.6 \%$ que opina lo contrario, así como si conocen el Plan de Acción Tutorial (PAT) de su centro, con una respuesta negativa de un $75.3 \%$.

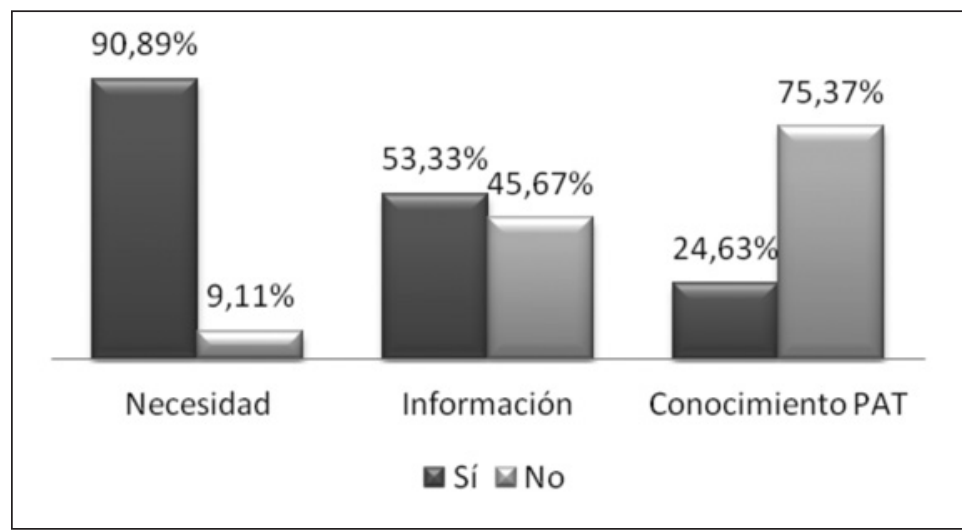

GRÁFICO 2

NECESIDAD E INFORMACIÓN SOBRE TUTORÍA. 
Estos datos inicialmente señalan que, a pesar de que la mayoría de los alumnos, independientemente de situarse en uno de los grupos, consideran la tutoría como necesaria en su proceso formativo, paradójicamente, casi la mitad de los estudiantes no disponen de información sobre la misma y la mayoría no conoce el documento de gestión institucional que rige la tutoría en su centro, es decir, el Plan de Acción Tutorial, aprobado por Junta de Facultad y presentado en los inicios del curso académico. Esto nos induce a pensar que la difusión que se le ha dado no ha sido suficiente ni adecuada y, como consecuencia, este desconocimiento puede llevar al alumnado a no beneficiarse de las virtualidades de este proceso.

Para este segundo objetivo, también nos interesa saber la información de la que disponen los participantes en relación a los servicios de orientación y asesoramiento de la UMU. Los resultados reflejan un alto desconocimiento por parte de los estudiantes en relación a todos ellos, aunque, a partir del análisis chi cuadrado, nos encontramos con ciertas diferencias significativas $(p<.05)$, tal y como puede apreciarse en la Tabla 3.

TABLA 3

CONOCIMIENTO DE LOS SERVICIOS DE ORIENTACIÓN Y ASESORAMIENTO DE LA UMU.

\begin{tabular}{cccccc}
\hline & & Nada & Regular & Mucho & $\begin{array}{c}\text { Chi cuadrado } \\
\text { Gl } \\
\text { Sig }\end{array}$ \\
\hline \multirow{2}{*}{ COIE } & Nuevo ingreso & $67.29 \%$ & $29.52 \%$ & $3.19 \%$ & 97.944 \\
& Veteranos & $26.96 \%$ & $59.13 \%$ & $13.91 \%$ & .000 \\
\hline \multirow{2}{*}{ SAOP } & Nuevo ingreso & $63.27 \%$ & $31.37 \%$ & $5.36 \%$ & 6.717 \\
& Veteranos & $53.48 \%$ & $41.74 \%$ & $4.78 \%$ & 2 \\
\hline \multirow{2}{*}{ SIU } & Nuevo ingreso & $34.49 \%$ & $51.60 \%$ & $13.90 \%$ & .035 \\
& Veteranos & $49.57 \%$ & $43.04 \%$ & $7.39 \%$ & 2 \\
\hline \multirow{2}{*}{ SRI } & Nuevo ingreso & $61.99 \%$ & $33.42 \%$ & $4.58 \%$ & .000 \\
\hline & Veteranos & $72.49 \%$ & $23.14 \%$ & $4.37 \%$ & 2 \\
\hline
\end{tabular}

En este sentido, los alumnos veteranos parecen conocer más el Centro de Orientación e Información de Empleo (COIE), con un $13.91 \%$ para los que opinan que mucho y un $59.13 \%$ para los que opinan que regular, frente a un $3.19 \%$ (mucho) y un $29.52 \%$ (regular) de los alumnos principiantes. Este hecho coincide con las necesidades que presenta el alumnado más antiguo, ya que está compuesto por estudiantes de $5^{\circ}$ curso que ven su inserción laboral mucho más cercana que los de $1^{\underline{0}}$. También son los alumnos veteranos los que parecen conocer mejor el Servicio de Asesoramiento y Orientación Personal (SAOP), con un 4.78\% (mucho) y un 41.74\% (regular) frente a un 5.36\% (mucho) y un 31.37\% (regular) para los estudiantes noveles. Esto también puede deberse a que este grupo de veteranos se compone 
además de estudiantes en el ecuador de su carrera, es decir, en $3^{\text {er }}$ curso, por lo que sus necesidades se corresponden con la oferta de este servicio, encaminado a propiciar el aprovechamiento de los estudios universitarios. Por su parte, los estudiantes de nuevo ingreso afirman conocer más el Servicio de Información Universitario (SIU), con un $13.9 \%$ (mucho) y un 51.6\% (regular). Este hecho es coherente con las necesidades de los alumnos de $1^{\circ}$, pues se asocian más con la información y, en este sentido, este servicio ha sido la primera referencia para formalizar la matrícula o resolver dudas de tipo administrativo.

Por último, también es interesante conocer para qué consideran importante la tutoría universitaria. En el Gráfico 3 se observa que tanto los alumnos veteranos como los de nuevo ingreso le conceden mucha importancia a las opciones Desarrollo académico (con un $56.57 \%$ y un $72.44 \%$, respectivamente) y Desarrollo profesional (con un $54.62 \%$ y un $63.91 \%$, respectivamente).

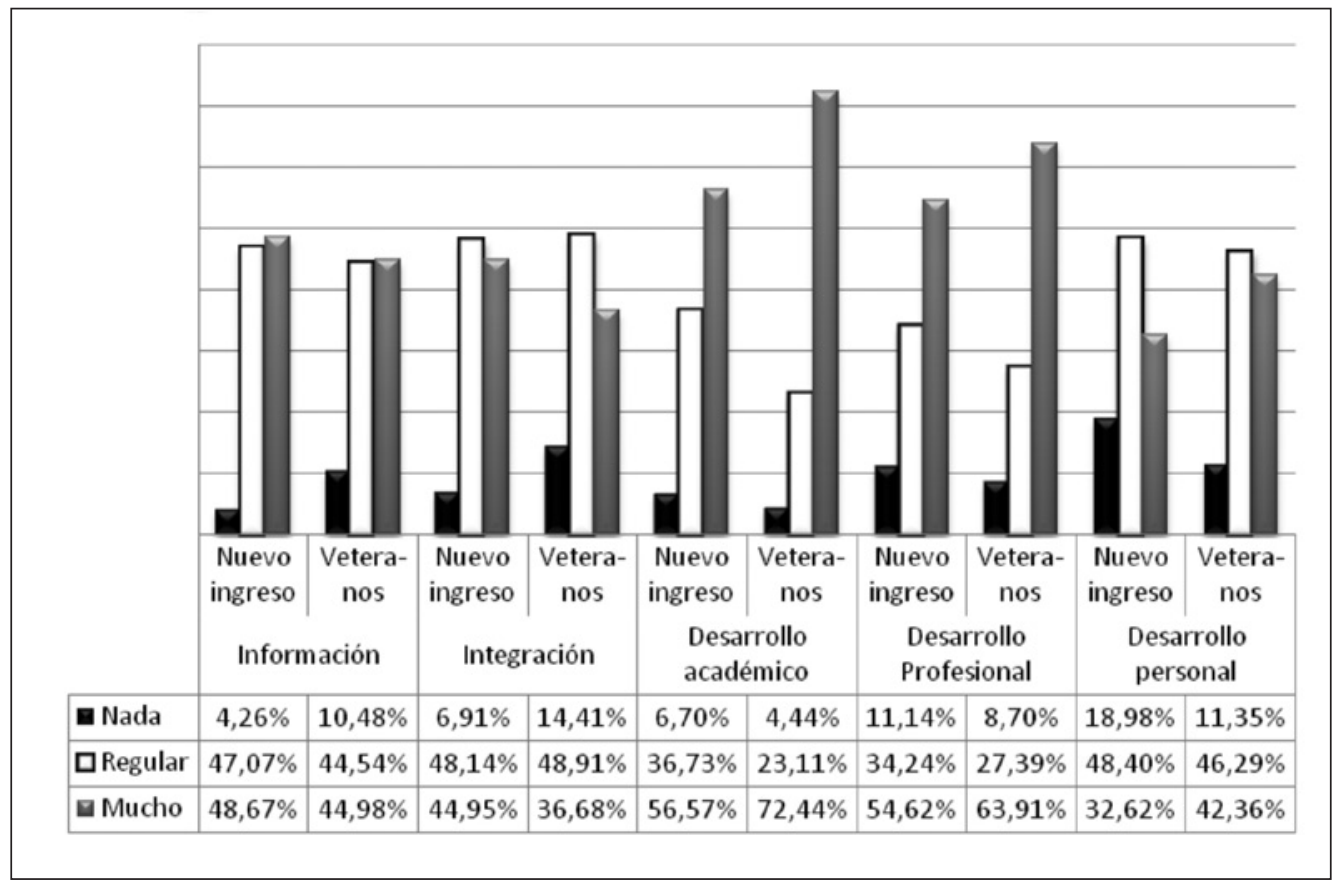

\section{GRÁFICO 3 \\ IMPORTANCIA DE LA TUTORÍA PARA LOS PARTICIPANTES.}

Estos resultados corroboran la respuesta anterior a favor de la tutoría como necesaria para el desarrollo de su proceso formativo, pero, a la vez, y tal vez por las experiencias vividas hasta ahora en relación a ésta, continúan viéndola desde una óptica un tanto reduccionista y restringida al ámbito académico.

De nuevo se aprecian diferencias significativas en casi todas las opciones de respuesta planteadas a los dos grupos de estudiantes, tal y como se muestra en la Tabla 4. 
TABLA 4

IMPORTANCIA DE LA TUTORÍA PARA LOS PARTICIPANTES. DIFERENCIAS SIGNIFICATIVAS.

\begin{tabular}{ccccc}
\hline & & $\begin{array}{c}\text { Chi } \\
\text { cuadrado }\end{array}$ & Gl & sig \\
\hline Información & $\begin{array}{c}\text { Nuevo ingreso } \\
\text { Veteranos }\end{array}$ & 8.950 & 2 & .011 \\
\hline Integración & $\begin{array}{c}\text { Nuevo ingreso } \\
\text { Veteranos }\end{array}$ & 10.542 & 2 & .005 \\
\hline $\begin{array}{c}\text { Desarrollo } \\
\text { académico }\end{array}$ & $\begin{array}{c}\text { Nuevo ingreso } \\
\text { Veteranos }\end{array}$ & 15.113 & 2 & .001 \\
\hline $\begin{array}{c}\text { Desarrollo } \\
\text { personal }\end{array}$ & $\begin{array}{c}\text { Nuevo ingreso } \\
\text { Veteranos }\end{array}$ & 8.981 & 2 & .011 \\
\hline
\end{tabular}

Los estudiantes principiantes valoran más la tutoría para obtener Información y para propiciar su Integración en el centro, mientras que valoran menos el Desarrollo académico y el Desarrollo personal. Estos resultados se corresponden con las necesidades que presentan estos alumnos, mucho más relacionadas con la adaptación e integración al centro.

Respecto al tercer objetivo (demandas a la tutoría y al tutor), los estudiantes solicitan a la tutoría universitaria Información (con un 73.6\% para los estudiantes principiantes y un $77.39 \%$ para el alumnado veterano) y Atención a alumnos con necesidad de apoyo educativo (con un $64.44 \%$ para alumnado de nuevo ingreso y un $67.69 \%$ para veteranos) (Tabla 5). Es curioso que en esta ocasión sean los alumnos más antiguos los que valoren en mayor medida la opción de Información, pues, como ya hemos visto anteriormente, le concedían menos importancia que los estudiantes principiantes, no siendo una de las opciones más valoradas, por lo que resulta paradójico que ahora sí se convierta en una de sus principales demandas.

También en esta ocasión el análisis mediante la prueba chi cuadrado refleja diferencias significativas en tres de las opciones de respuesta planteadas (Tabla 5). De esta manera, los estudiantes de nuevo ingreso demandan en mayor medida (46.52\%) que los veteranos (38.16\%) la Resolución de conflictos, tal vez por la utilización por parte del profesorado, cada vez con más frecuencia, de nuevas metodologías relacionadas con el trabajo cooperativo. En cambio, los estudiantes veteranos lo que demandan a la tutoría es la Ayuda en las transiciones y la Toma de decisiones, respuestas coherentes con el momento formativo en el que se encuentran estos alumnos, finalizando los estudios, como ya hemos comentado.

En este tercer objetivo también se pregunta por las competencias profesionales que demandan a sus tutores y, como se muestra en el Gráfico 4, todo el alumnado participante le concede mucha importancia a todas las competencias propuestas. 


\section{TABLA 5}

DEMANDAS DE LOS PARTICIPANTES A LA TUTORÍA UNIVERSITARIA.

\begin{tabular}{|c|c|c|c|c|c|}
\hline & & Nada & Regular & Mucho & $\begin{array}{c}\text { Chi cuadrado } \\
\text { Gl } \\
\text { Sig }\end{array}$ \\
\hline \multirow{3}{*}{ Información } & Nuevo ingreso & $2.93 \%$ & $23.47 \%$ & $73.60 \%$ & 2.186 \\
\hline & & & & & 2 \\
\hline & Veteranos & $3.91 \%$ & $18.70 \%$ & $77.39 \%$ & .335 \\
\hline \multirow{2}{*}{$\begin{array}{l}\text { Seguimiento } \\
\text { académico }\end{array}$} & Nuevo ingreso & $5.85 \%$ & $47.34 \%$ & $46.81 \%$ & 3.118 \\
\hline & Veteranos & $6.52 \%$ & $40.00 \%$ & $53.48 \%$ & .210 \\
\hline \multirow{2}{*}{$\begin{array}{c}\text { Orientación } \\
\text { itinerario formativo }\end{array}$} & Nuevo ingreso & $4.30 \%$ & $33.60 \%$ & $62.10 \%$ & 4.408 \\
\hline & Veteranos & $6.58 \%$ & $26.32 \%$ & $67.11 \%$ & .110 \\
\hline \multirow{2}{*}{$\begin{array}{l}\text { Inserción socio } \\
\text { laboral }\end{array}$} & Nuevo ingreso & $6.93 \%$ & $34.40 \%$ & $58.67 \%$ & 3.714 \\
\hline & Veteranos & $8.26 \%$ & $26.96 \%$ & $64.78 \%$ & .156 \\
\hline \multirow{2}{*}{$\begin{array}{l}\text { Orientación } \\
\text { personal }\end{array}$} & Nuevo ingreso & $8.82 \%$ & $43.05 \%$ & $48.13 \%$ & .891 \\
\hline & Veteranos & $10.04 \%$ & $39.30 \%$ & $50.66 \%$ & .640 \\
\hline \multirow{2}{*}{$\begin{array}{l}\text { Resolución } \\
\text { conflictos }\end{array}$} & Nuevo ingreso & $8.56 \%$ & $44.92 \%$ & $46.52 \%$ & 6.543 \\
\hline & Veteranos & $14.04 \%$ & $47.81 \%$ & $38.16 \%$ & .038 \\
\hline \multirow{3}{*}{ Toma de decisiones } & Nuevo ingreso & $13.07 \%$ & $55.20 \%$ & $31.73 \%$ & 7.668 \\
\hline & & & & & 2 \\
\hline & Veteranos & $12.72 \%$ & $44.74 \%$ & $42.54 \%$ & .022 \\
\hline \multirow{2}{*}{$\begin{array}{l}\text { Ayuda en } \\
\text { transiciones }\end{array}$} & Nuevo ingreso & $11.96 \%$ & $52.17 \%$ & $35.87 \%$ & 9.775 \\
\hline & Veteranos & $17.03 \%$ & $39.30 \%$ & $43.67 \%$ & .008 \\
\hline \multirow{2}{*}{$\begin{array}{l}\text { Atención a alumnos } \\
\text { con necesidad de } \\
\text { apoyo educativo }\end{array}$} & Nuevo ingreso & $7.22 \%$ & $28.34 \%$ & $64.44 \%$ & $\begin{array}{c}1.860 \\
2\end{array}$ \\
\hline & Veteranos & $8.73 \%$ & $23.58 \%$ & $67.69 \%$ & .395 \\
\hline
\end{tabular}




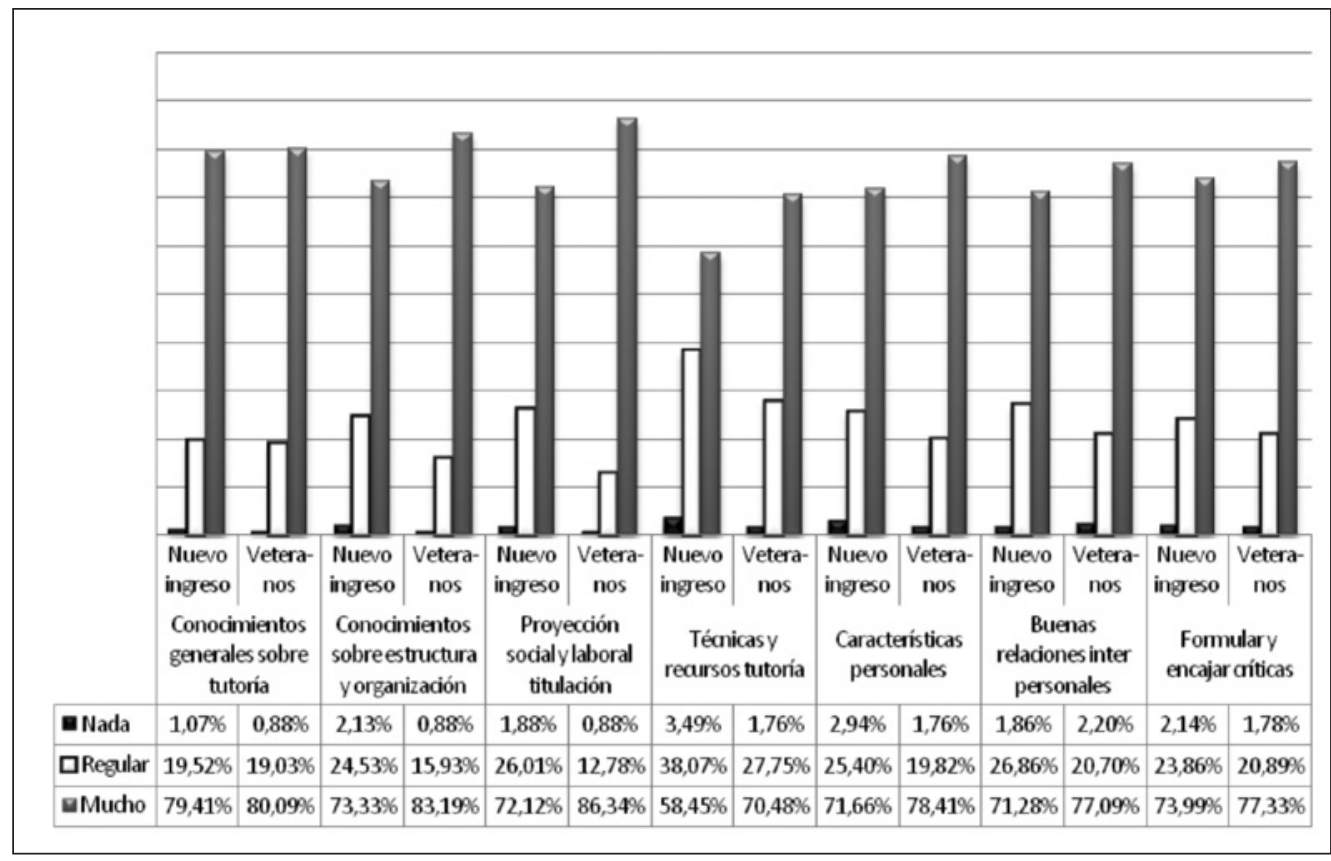

\section{GRÁFICO 4}

COMPETENCIAS PROFESIONALES DEL TUTOR DEMANDADAS POR LOS PARTICIPANTES.

En la Tabla 6 se muestran las diferencias significativas que se obtienen a partir de la prueba chi cuadrado, apreciándose diferencias en tres de las opciones planteadas. En general, el alumando veterano reclama a su tutor Conocimientos sobre la estructura y organización de la titulación, así como de la UMU en general (servicios, ofertas de actividades, etc.). Esta respuesta sorprende, ya que parece atender más a necesidades de estudiantes de nuevo ingreso, pero, por otra parte, refleja un gran desconocimiento de nuestros alumnos en relación a la propia institución, a pesar de encontrarse en cursos avanzados.

TABLA 6

COMPETENCIAS PROFESIONALES DEL TUTOR DEMANDADAS POR LOS PARTICIPANTES. DIFERENCIAS SIGNIFICATIVAS.

\begin{tabular}{lcccc}
\hline & Chi cuadrado & Gl & Sig \\
\hline $\begin{array}{l}\text { Conocimientos sobre } \\
\text { estructura y organización }\end{array}$ & $\begin{array}{c}\text { Nuevo ingreso } \\
\text { Veteranos }\end{array}$ & 7.999 & 2 & .018 \\
\hline $\begin{array}{l}\text { Proyección social y laboral } \\
\text { titulación }\end{array}$ & $\begin{array}{c}\text { Nuevo ingreso } \\
\text { Veteranos }\end{array}$ & 16.380 & 2 & .000 \\
\hline Técnicas y recursos tutoría & $\begin{array}{c}\text { Nuevo ingreso } \\
\text { Veteranos }\end{array}$ & 9.122 & 2 & .010 \\
\hline
\end{tabular}


Igualmente, este grupo de estudiantes también demanda a sus tutores Conocimientos sobre la proyección social y profesional de la titulación. En esta ocasión, la respuesta es coherente dado que se trata de alumnos que bien se encuentran en el ecuador de su carrera o a punto de terminar sus estudios universitarios, por lo que empiezan a preocuparse por la proyección de los mismos.

La última opción en la que se aprecian diferencias significativas entre los grupos de estudio es en la de Aplicación de técnicas y recursos propios de la tutoría ( $p=.010)$. De nuevo, son los estudiantes veteranos los que también le conceden mayor valor a esta alternativa, a pesar de no gozar de una figura que, al igual que ocurre con los estudiantes de nuevo ingreso, les guíe en su proceso formativo, pues es a partir de los títulos de Grado cuando se empieza a reconocer la figura del tutor universitario.

En relación al cuarto objetivo (Identificar qué tipo de tutoría utilizan los estudiantes y comparar para qué hacen uso de ella), el alumnado, en términos generales, afirma utilizar con mayor frecuencia la tutoría virtual, seguida de la personal. Este hecho, que podemos observar en el Gráfico 5, indica el auge que han alcanzado las TICs, ya que permite una comunicación mucho más directa y rápida, además de no supeditar esa comunicación al tiempo y al espacio.

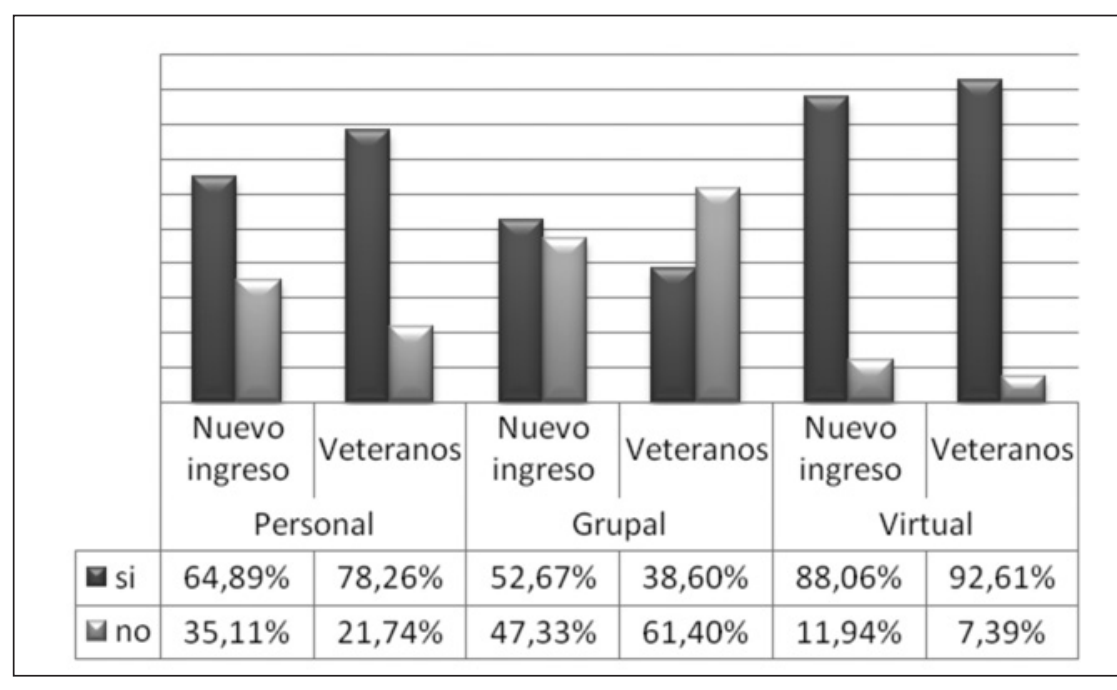

GRÁFICO 5

UTILIZACIÓN DE DIFERENTES TIPOS DE TUTORÍA.

En la Tabla 7 se ofrecen las diferencias significativas, obtenidas a partir de la prueba chi cuadrado, entre los dos grupos en relación a la utilización de la tutoría personal y la grupal. Son los estudiantes veteranos los que afirman utilizar con mayor frecuencia (78.26\%) la personal, en comparación con el grupo de nuevo ingreso $(64.89 \%)$, mientras que son éstos los que afirman utilizar con mayor frecuencia la grupal (52.67\%), frente a los más veteranos (38.6\%). La respuesta es coherente y ajustada, pues en los planes de estudio de Licenciatura y Diplomatura no está extendida, o generalizada, 
la realización de tutorías grupales dentro de las distintas asignaturas, así como tampoco la asignación de un profesor para que tutele a un grupo determinado de alumnos durante un periodo de tiempo más o menos largo y que, de esta manera, pueda tratar con ellos diferentes temáticas de interés común. En términos generales, los alumnos veteranos están más acostumbrados a dirigirse de manera personal, directa e individual al profesor correspondiente para la resolución de sus dudas. Por el contrario, para el grupo de principiantes esta práctica de tutoría grupal es algo totalmente normal, sobre todo en el caso de nuestro centro, ya que a los tutores se les asigna un determinado grupo-clase compuesto por una media de 80 alumnos, lo que invita al uso de este tipo de tutoría.

TABLA 7

UTILIZACIÓN DE DIFERENTES TIPOS DE TUTORÍA. DIFERENCIAS SIGNIFICATIVAS.

\begin{tabular}{lcccc}
\hline & & Chi cuadrado & Gl & Sig \\
\hline Personal & $\begin{array}{c}\text { Nuevo ingreso } \\
\text { Veteranos }\end{array}$ & 12.135 & 1 & .000 \\
\hline Grupal & $\begin{array}{c}\text { Nuevo ingreso } \\
\text { Veteranos }\end{array}$ & 11.260 & 1 & .001 \\
\hline
\end{tabular}

Por otra parte, si nos detenemos a observar para qué hacen uso de la tutoría personal, se aprecia en el Grafico 6 y en la Tabla 8 que ambos grupos la utilizan para solicitar Información y realizar un Seguimiento académico de las asignaturas correspondientes. Para el resto de las opciones de respuesta ofrecidas se aprecia un índice de respuesta muy bajo, que ratifica la concepción academicista de la tutoría por parte de los participantes.

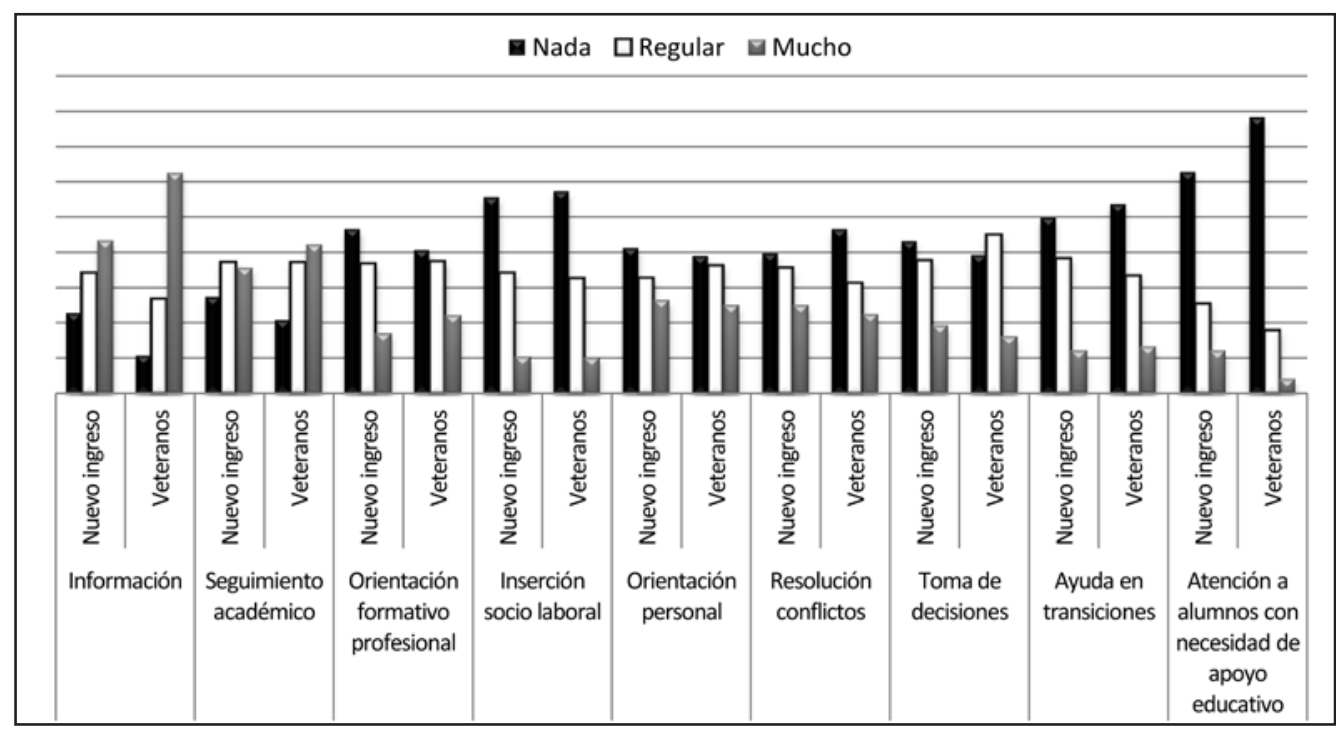

GRÁFICO 6

USO DE LA TUTORÍA PERSONAL. 
TABLA 8

USO DE LA TUTORÍA PERSONAL.

\begin{tabular}{cccccc}
\hline & & & & Mucho & $\begin{array}{c}\text { Chi } \\
\text { cuadrado } \\
\text { Gl }\end{array}$ \\
Sig
\end{tabular}

Las diferencias significativas, a partir de la prueba chi cuadrado ( $p<.05)$, se encuentran en las alternativas de Información $(p=.000)$ y Atención a alumnos con necesidad de apoyo educativo ( $p=.001$ ). Una vez más apreciamos que los alumnos veteranos hacen uso de la tutoría personal para obtener Información, con un $62.5 \%$ de respuesta frente al $43.1 \%$ de los estudiantes noveles. Este hecho es curioso y contradictorio, ya que anteriormente no le concedían importancia a la tutoría en este sentido, aunque esta respuesta sí se corresponde con las demandas, vistas anteriormente.

Consideramos que, dado el momento formativo en el que se encuentran, la tutoría podría ofrecer a estos alumnos muchas más oportunidades que las que se les proporciona desde el horario de atención a alumnos de cada asignatura. Por otra parte, son los alumnos de nuevo ingreso los que usan más la tutoría personal para la Atención a alumnos con necesidad de apoyo educativo, aunque no en un alto porcentaje (11.84\%). 
Esta respuesta es afín al nuevo escenario europeo que concibe la tutoría firmemente asentada en la atención a la diversidad del alumnado con la intención de contribuir a la personalización de su proceso de aprendizaje tal y como apuesta el Estatuto del Estudiante Universitario (Real Decreto 1791/2010, de 30 de diciembre).

En cuanto al uso que hacen los participantes de la tutoría grupal (Gráfico 7), se vuelve a apreciar que los dos grupos la utilizan para obtener Información, mientras que el resto de opciones de respuesta son escasamente valoradas, como ocurría anteriormente.

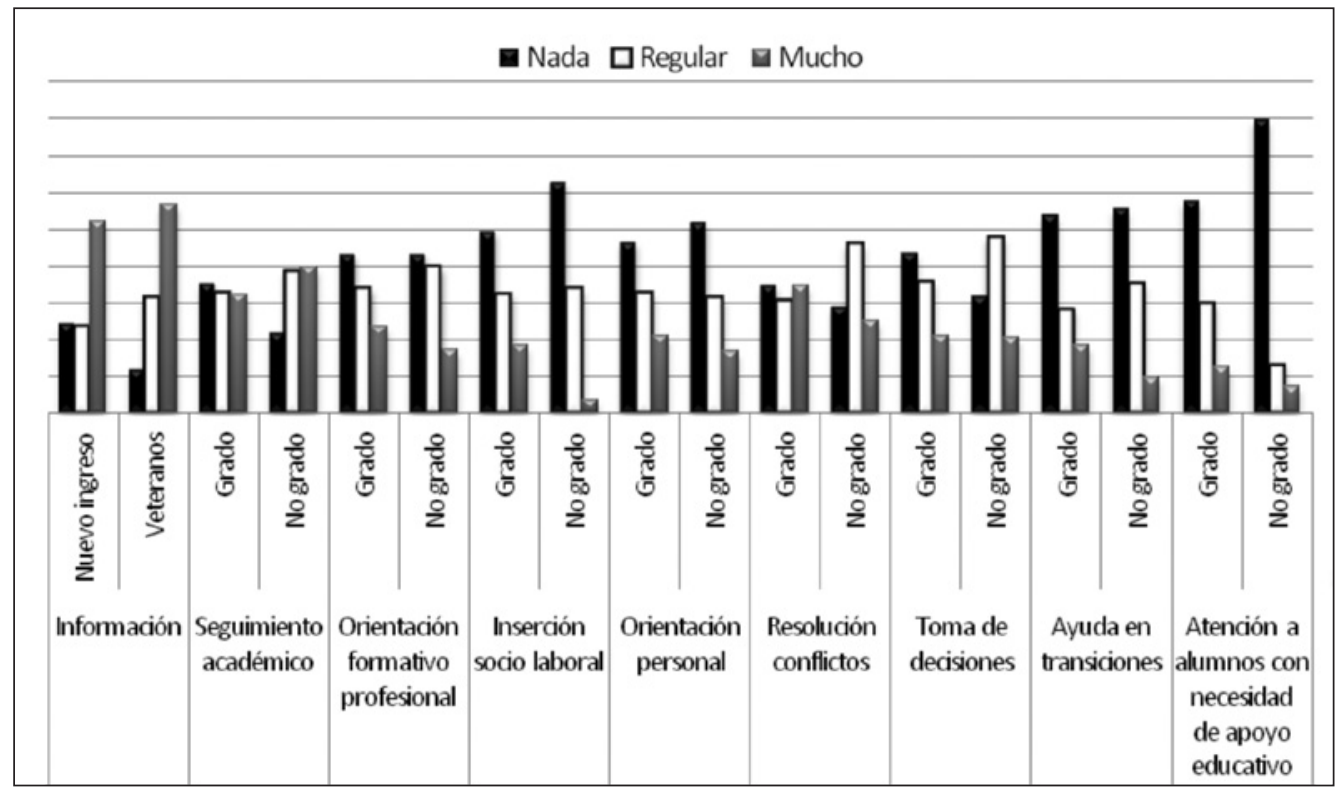

\section{GRÁFICO 7 \\ USO DE LA TUTORÍA GRUPAL.}

En la Tabla 9 se muestran las diferencias significativas en las distintas opciones de respuesta y se aprecia que en la alternativa Inserción socio-laboral $(p=.004)$, paradógicamente, son los alumnos noveles los que afirman utilizarla con mucha frecuencia $(18.28 \%)$, a pesar de tratarse de estudiantes de nuevo ingreso. Aunque no se trate de un porcentaje mayoritario, es interesante observar que los alumnos de primer curso tratan ya este tipo de temáticas, pues si concebimos la tutoría como un proceso continuo, integral e integrador, no es necesario profundizar en ellas únicamente cuando los estudiantes se encuentran en los últimos años de su formación. Para la opción Resolución de conflictos ( $p=.049$ ) también es este grupo quien afirma hacer uso de la tutoría grupal para esta finalidad, quizás por la utilizacion de metodologias activas por parte del profesorado, metodologías que implican trabajo colaborativo y cooperativo, lo que puede traer como consecuencia mayores desavenencias entre los miembros de un grupo-clase. Para la opción de Atención a alumnos con necesidad de apoyo educativo $(p=.002)$ vuelven a ser los alumnos principiantes los que más uso hacen de ella, por las razones argumentadas anteriormente. 
TABLA 9

USO DE LA TUTORÍA GRUPAL.

\begin{tabular}{|c|c|c|c|c|c|}
\hline & & Nada & Regular & Mucho & $\begin{array}{c}\text { Chi cuadrado } \\
\text { Gl } \\
\text { Sig }\end{array}$ \\
\hline \multirow[t]{2}{*}{ Información } & Nuevo ingreso & $24.19 \%$ & $23.66 \%$ & $52.15 \%$ & $\begin{array}{c}5.652 \\
2\end{array}$ \\
\hline & Veteranos & $12.05 \%$ & $31.33 \%$ & $56.63 \%$ & .059 \\
\hline \multirow{2}{*}{$\begin{array}{l}\text { Seguimiento } \\
\text { académico }\end{array}$} & Nuevo ingreso & $35.29 \%$ & $32.62 \%$ & $32.09 \%$ & 4.992 \\
\hline & Veteranos & $21.69 \%$ & $38.55 \%$ & $39.76 \%$ & .082 \\
\hline \multirow{2}{*}{$\begin{array}{c}\text { Orientación } \\
\text { itinerario formativo }\end{array}$} & Nuevo ingreso & $42.93 \%$ & $33.70 \%$ & $23.37 \%$ & 1.505 \\
\hline & Veteranos & $43.21 \%$ & $39.51 \%$ & $17.28 \%$ & .471 \\
\hline \multirow{2}{*}{$\begin{array}{l}\text { Inserción socio- } \\
\text { laboral }\end{array}$} & Nuevo ingreso & $49.46 \%$ & $32.26 \%$ & $18.28 \%$ & 10.876 \\
\hline & Veteranos & $62.65 \%$ & $33.73 \%$ & $3.61 \%$ & .004 \\
\hline \multirow{2}{*}{$\begin{array}{c}\text { Orientación } \\
\text { personal }\end{array}$} & Nuevo ingreso & $46.49 \%$ & $32.43 \%$ & $21.08 \%$ & .873 \\
\hline & Veteranos & $51.81 \%$ & $31.33 \%$ & $16.87 \%$ & .646 \\
\hline \multirow{2}{*}{$\begin{array}{l}\text { Resolución } \\
\text { conflictos }\end{array}$} & Nuevo ingreso & $34.76 \%$ & $30.48 \%$ & $34.76 \%$ & 6.035 \\
\hline & Veteranos & $28.92 \%$ & $45.78 \%$ & $25.30 \%$ & .049 \\
\hline \multirow{3}{*}{ Toma de decisiones } & Nuevo ingreso & $43.55 \%$ & $35.48 \%$ & $20.97 \%$ & 4.119 \\
\hline & & & & & 2 \\
\hline & Veteranos & $31.71 \%$ & $47.56 \%$ & $20.73 \%$ & .128 \\
\hline \multirow{2}{*}{$\begin{array}{l}\text { Ayuda en } \\
\text { transiciones }\end{array}$} & Nuevo ingreso & $53.76 \%$ & $27.96 \%$ & $18.28 \%$ & 3.703 \\
\hline & Veteranos & $55.42 \%$ & $34.94 \%$ & $9.64 \%$ & .157 \\
\hline \multirow{2}{*}{$\begin{array}{l}\text { Atención a alumnos } \\
\text { con necesidad de } \\
\text { apoyo educativo }\end{array}$} & Nuevo ingreso & $57.69 \%$ & $29.67 \%$ & $12.64 \%$ & 12.382 \\
\hline & Veteranos & $79.76 \%$ & $13.10 \%$ & $7.14 \%$ & .002 \\
\hline
\end{tabular}

En relación a la tutoría virtual (Grafico 8), se observa el mismo patrón de respuesta que el dado para la tutoría personal y grupal. Los dos grupos de alumnos afirman usar la tutoría virtual para obtener Información y para realizar el Seguimiento académico de las distintas asignaturas. El resto de las opciones de respuesta continúan siendo muy poco valoradas. 


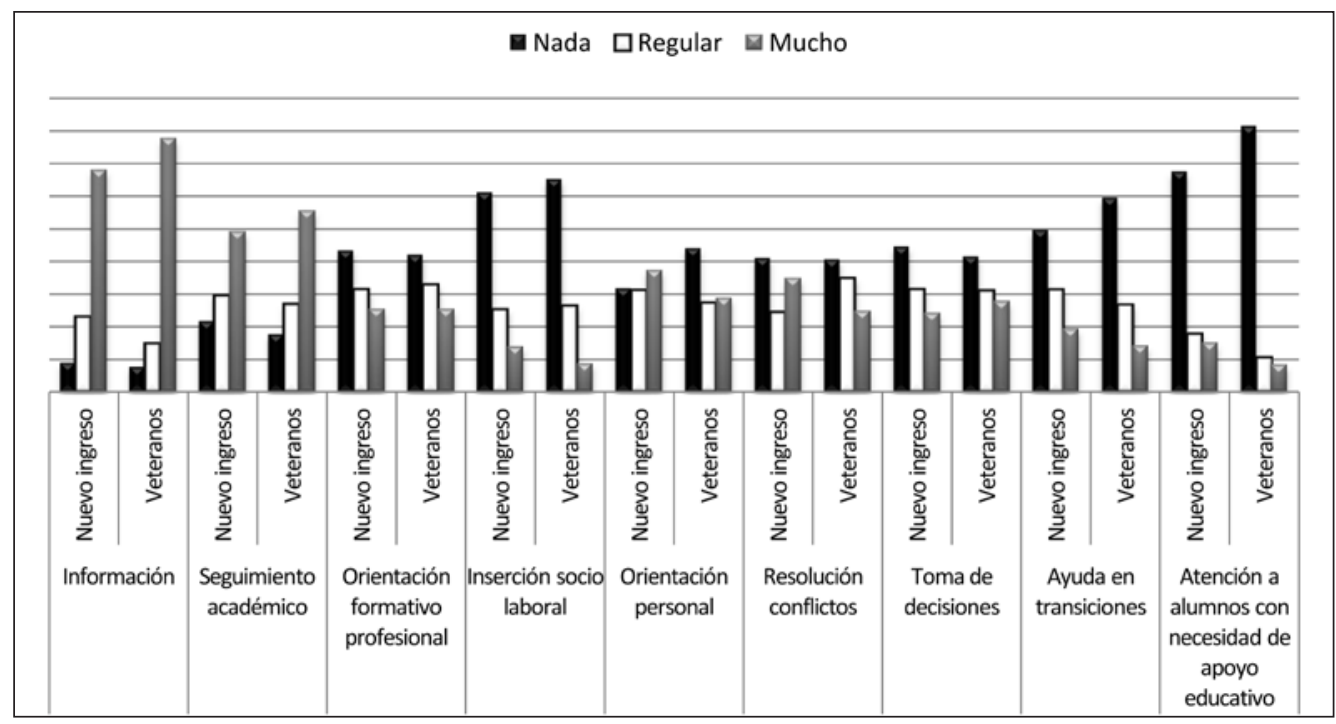

\section{GRÁFICO 8 \\ USO DE LA TUTORÍA VIRTUAL.}

La Tabla 10 muestra las diferencias significativas en las opciones Información ( $p=.043$ ), Orientación personal ( $p=.013)$, Resolución de conflictos $(p=.012)$ y Atención a alumnos con necesidad de apoyo educativo ( $p=.002)$.

TABLA 10

USO DE LA TUTORÍA VIRTUAL.

\begin{tabular}{cccccc}
\hline & & & & & Chi cuadrado \\
& & Nada & Regular & Mucho & Sig \\
\hline \multirow{2}{*}{ Información } & Nuevo ingreso & $9.01 \%$ & $22.98 \%$ & $68.01 \%$ & 6.274 \\
& Veteranos & $7.62 \%$ & $14.76 \%$ & $77.62 \%$ & .043 \\
\hline $\begin{array}{c}\text { Seguimiento } \\
\text { académico }\end{array}$ & Nuevo ingreso & $21.63 \%$ & $29.47 \%$ & $48.90 \%$ & 2.355 \\
\hline $\begin{array}{c}\text { Orientación } \\
\text { itinerario } \\
\text { formativo }\end{array}$ & Vueteranos & $17.70 \%$ & $26.79 \%$ & $55.50 \%$ & .308 \\
\hline $\begin{array}{c}\text { Inserción socio- } \\
\text { laboral }\end{array}$ & Nuevo ingreso & $43.26 \%$ & $31.35 \%$ & $25.39 \%$ & .146 \\
\hline $\begin{array}{c}\text { Orientación } \\
\text { personal }\end{array}$ & Veteranos & $61.06 \%$ & $25.23 \%$ & $13.71 \%$ & 3.192 \\
\hline
\end{tabular}




\begin{tabular}{|c|c|c|c|c|c|}
\hline & & Nada & Regular & Mucho & $\begin{array}{c}\text { Chi cuadrado } \\
\text { Gl } \\
\text { Sig }\end{array}$ \\
\hline \multirow{2}{*}{$\begin{array}{l}\text { Resolución } \\
\text { conflictos }\end{array}$} & Nuevo ingreso & $40.94 \%$ & $24.38 \%$ & $34.69 \%$ & $\begin{array}{c}8.870 \\
2\end{array}$ \\
\hline & Veteranos & $40.48 \%$ & $34.76 \%$ & $24.76 \%$ & .012 \\
\hline \multirow{2}{*}{$\begin{array}{c}\text { Toma de } \\
\text { decisiones }\end{array}$} & Nuevo ingreso & $44.51 \%$ & $31.35 \%$ & $24.14 \%$ & $\begin{array}{c}.886 \\
2\end{array}$ \\
\hline & Veteranos & $41.43 \%$ & $30.95 \%$ & $27.62 \%$ & .642 \\
\hline \multirow{2}{*}{$\begin{array}{c}\text { Ayuda en } \\
\text { transiciones }\end{array}$} & Nuevo ingreso & $49.52 \%$ & $31.31 \%$ & $19.17 \%$ & 5.173 \\
\hline & Veteranos & $59.42 \%$ & $26.57 \%$ & $14.01 \%$ & .075 \\
\hline \multirow{2}{*}{$\begin{array}{l}\text { Atención a } \\
\text { alumnos con } \\
\text { necesidad de } \\
\text { apoyo educativo }\end{array}$} & Nuevo ingreso & $67.41 \%$ & $17.72 \%$ & $14.87 \%$ & 12.419 \\
\hline & Veteranos & $81.34 \%$ & $10.53 \%$ & $8.13 \%$ & $\begin{array}{c}2 \\
.002\end{array}$ \\
\hline
\end{tabular}

En el caso de la alternativa Información continúan siendo los alumnos veteranos $(77.62 \%)$ los que afirman hacer uso de este tipo de tutoría para esta finalidad, a pesar de que, bajo nuestro punto de vista, se trata mas de una necesidad de estudiantes de nuevo ingreso. Para el resto de opciones es el grupo de nuevo ingreso el que hace uso de todas ellas con mayor frecuencia, lo que indica que, en cierta medida, conforme el alumnado pasa más tiempo en la comunidad uniersitaria, va abandonando la idea academicista de la tutoría para contemplar e integrar otros aspectos que influirán en su desarrollo y formacion integral.

Finalmente, para el quinto objetivo se plantea abiertamente a los participantes qué propuestas realizarían para mejorar la puesta en marcha de la tutoría universitaria para cada uno de los tipos de tutoría analizados (presencial, grupal y virtual). A esta pregunta responde el $36.07 \%$ de los participantes y las categorías establecidas tras el análisis de contenido realizado se resumen en: Implicación y cumplimiento, Horarios, Obligatoriedad/Frecuencia, Competencias del tutor e Información, distribuyéndose tal y como puede apreciarse en el Gráfico 9.

Se observa que el mayor número de propuestas, con una gran diferencia $(81.8 \%)$, se dirigen a la tutoría virtual y, en concreto, dentro de la categoría de Implicación y cumplimiento, debido, tal y como podemos deducir de las respuestas de los participantes, a la escasa respuesta del profesorado. Dentro de esta misma categoría, otro bloque de propuestas se concentran en la tutoría grupal (27.1\%), con la intención de que se produzca un mayor desarrollo de la misma. También es interesante señalar que las propuestas encaminadas a mejorar el desarrollo de la tutoría personal se sitúan dentro de la categoría de Información e Implicación y cumplimiento (con un 25.9\% y un $25.3 \%$, respectivamente). Estos resultados nos indican que el alumnado tiene ante todo una falta de información y sienten que el profesorado no se compromete con la función tutorial. 


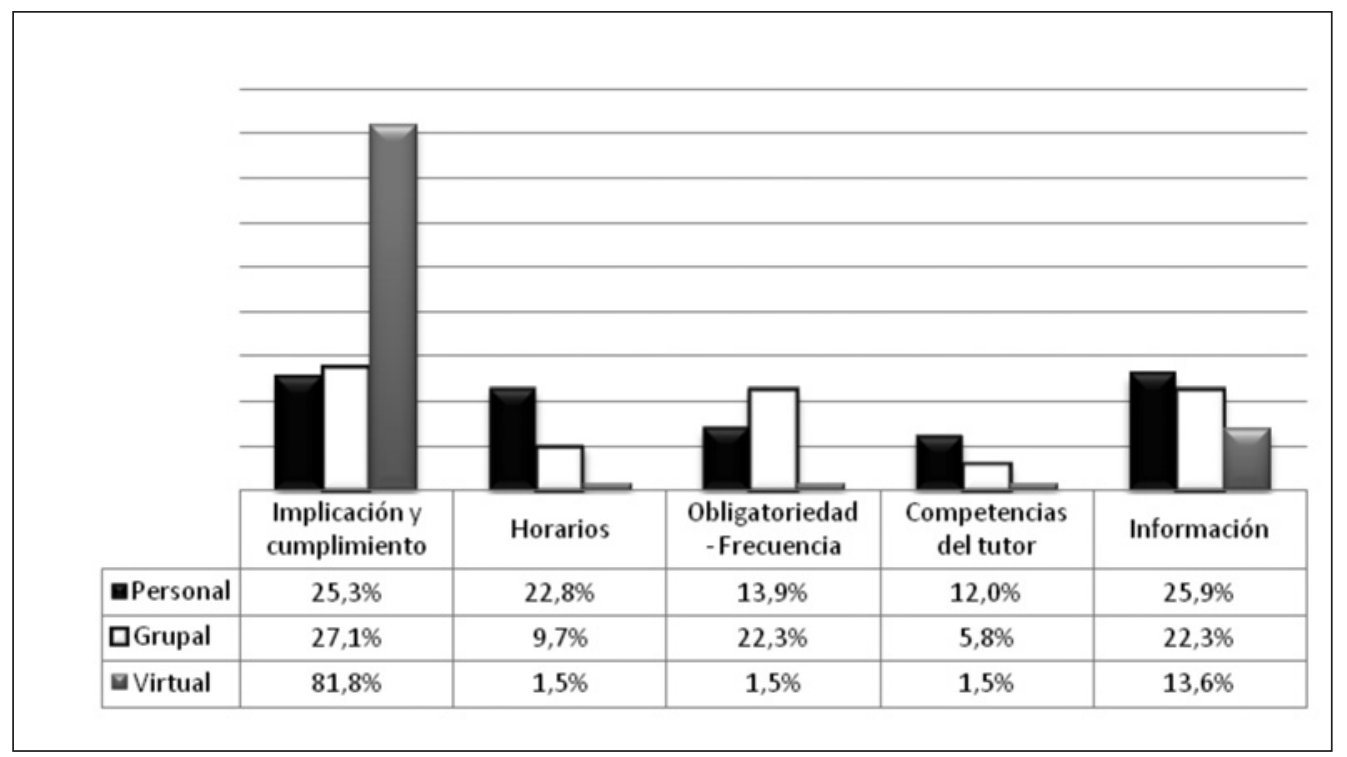

\section{GRÁFICO 9 \\ PROPUESTAS DE MEJORA PARA EL BUEN DESARROLLO DE LA TUTORÍA.}

La categoría Implicación y cumplimiento incluye propuestas relacionadas con la necesidad de un mayor compromiso por parte de los tutores y un mayor cumplimiento de los horarios de atención presencial; también con la necesidad de que el profesorado conteste a las preguntas que se les plantean vía correo electrónico u otros medios virtuales. Una de las quejas más habituales es la del incumplimiento de los horarios de atención por parte de los tutores; en este sentido, se expresa textualmente "que todo el profesorado tenga horario de tutorías tanto presencial como virtual y que lo cumplan, ya que muchas veces vas al despacho del profesor y no está".

La categoría de Horarios incluye las respuestas referidas a la necesidad de pactar o flexibilizar los horarios de atención de los tutores, con el objetivo de facilitar la asistencia del alumnado. Hay estudiantes que proponen que "las tutorías individuales no tengan lugar en horas de clase, puesto que me obliga a no asistir para ir a una tutoría presencial".

La categoría de Obligatoriedad-Frecuencia hace referencia a establecer de forma obligatoria y con cierta periodicidad tutorías con los alumnos, bien de manera individual, grupal y/o virtual. En este sentido, se trataría de que cada tutor estableciera un calendario tutorial para llevar a cabo con su grupo de alumnos a lo largo del curso académico.

Otro grupo de propuestas se categoriza como Competencias del tutor que incluye la capacidad del tutor de generar un buen ambiente, ponerse en el lugar del otro o intentar comprender a la persona.

Finalmente, la categoría Información hace referencia a disponer de mayor información y conocimiento acerca de las mismas, es decir, cuál es su finalidad, qué temas pueden ser tratados, qué objetivos se pretenden alcanzar con su realización, etc. 


\section{CONCLUSIONES}

Actualmente la universidad debe ir más allá de la función clásica de transmisión de conocimiento y de capacitación, reconsiderando que debe dar respuesta a la formación integral y ello comporta la necesidad de formar a personas capaces de desempeñar no sólo un rol profesional, sino también un rol en la sociedad en la que conviven. Esta idea es crecidamente aceptada por organismos nacionales, europeos e internacionales y la defienden muchos analistas y autores, entre los que destacamos a Lázaro (1997), Hernández y Domínguez (1998), Martínez, Buxarrais y Esteban (2002), Rodríguez Moreno (2002), Zabalza (2003), Pino y Ricoy (2006) y González Maura y González Tirados (2007).

En este escenario, la tutoría universitaria se concibe como un proceso orientador de carácter formativo que facilita este desarrollo y formación integral en su proyección académica, profesional y personal. Se trata de acompañar al estudiante a lo largo de su aprendizaje, con la intención de potenciar y favorecer su proyecto profesional y de vida.

Desde esta concepción la tutoría universitaria se construye más allá de la mera resolución de problemas puntuales y concretos, ya que comporta una relación de apoyo y ayuda entre profesor-tutor y alumno, que debe hacerse extensiva a toda la dinámica que conforma el proceso educativo. Debemos abandonar la concepción puramente burocrática de la tutoría por otra más amplia, más comprensiva, que vaya más allá del quehacer de todo profesor universitario en el desarrollo de sus asignaturas y que abarque aspectos relacionados con la madurez y el desarrollo integral del alumnado. Defendemos por tanto "una tutoría cuyo ámbito de actuación no se restringe puramente [...] al horario de las 6 horas y al espacio del despacho [...], sino que se extiende más allá, haciendo un seguimiento del proceso formativo y estimulando la madurez personal y profesional, con una clara proyección hacia el aprendizaje autónomo, la investigación y el desempeño profesional", tal y como señala Álvarez Pérez (2005-2006, p. 284).

Esta redefinición de la tutoría conlleva nuevas responsabilidades y modos de hacer que exigen la implicación de la institución universitaria a todos sus niveles. Actualmente, hay una posición bastante consensuada de tratar y afrontar desde la tutoría todos los aspectos que afecten a la globalidad del estudiante, sin embargo, tal y como hemos comprobado en nuestra investigación, existe una tendencia a asimilar la tutoría casi exclusivamente con la orientación puramente académica, pues se vincula a aspectos vinculados a la materia que imparte el profesor, relacionados esencialmente con la resolución de dudas antes de los exámenes y después para la revisión de los mismos, tal y como se manifiesta en otros estudios e investigaciones como los de Lázaro (1997, 2002), Sebastián y Sánchez (1999), Alañón (2000), Alcón (2003), Lobato, Arbizu y Castillo (2004), Arbizu, Lobato y Castillo (2005), García Nieto et al. (2004, 2005), Cid y Pérez (2006), Pérez Abellás (2006) y Zabalza y Cid (2006), entre otros.

Igualmente concluimos que nuestros estudiantes sí consideran necesaria e importante la tutoría para su formación universitaria, como demuestran numerosos trabajos entre los que destacamos los de Sebastián y Sánchez (1999), Rodicio, Villayandre y Pérez (2001), Lobato y Arbizu (2003), García Nieto et al. (2004, 2005) y Rumbo y Gómez (2009, 2011). Sin embargo, les falta información sobre ésta en todos los sentidos, independientemente de que se trate de alumnado de nuevo ingreso o veterano. A este respecto, 
debemos plantearnos que algo está fallando, si los principales destinatarios del PAT de un centro apenas lo conocen.

Constatamos como estas necesidades de información son diferentes según el momento en que se encuentre el alumno. Autores como Adame (2002), García Nieto et al. (2004), Hernández y Torres (2005), Álvarez, Asensio, Forner y Sobrado (2006), Gallego (2006) y Sobrado (2008), hacen una diferenciación respecto a las funciones que desarrolla un tutor según el momento o etapa de desarrollo, distinguiendo entre: el momento de entrada en la universidad, durante la estancia en ella y al finalizar sus estudios y prepararse para su inserción laboral.

Evidenciamos como nuestros estudiantes realizan demandas a la tutoría que responden a sus necesidades, las cuales se van transformado a medida que avanzan en su proceso formativo, como se muestra en los estudios anteriores, pero, sin embargo, desconocen el abanico de posibilidades que les ofrece la tutoría, reduciéndola a aspectos informativos y académicos, sobre todo, si no han experimentado otro modo de hacer tutoría. Estos resultados no coinciden con los obtenidos en otros estudios como los de Lázaro (1997, 2002, 2003), Álvarez Pérez (2002), Zabalza (2003), Gairín, Feixas, Guillamón y Quinquer (2004), Lobato, Arbizu y Castillo (2004), García Nieto et al. (2005) y Rodríguez Espinar (2008), entre otros, en donde ésta se relaciona con diferentes aspectos, no sólo con lo académico y lo administrativo, sino también con lo personal, lo social y lo profesional.

Respecto a la modalidad de tutoría más útil para los estudiantes, en coincidencia con otros estudios, como el de Martínez y Crespo (2009), en los que sobresale la utilización de la tutoría virtual básicamente por comodidad, ahorro de tiempo y rapidez, los estudiantes que participan en esta investigación afirman preferir también en primer lugar la tutoría virtual, seguida de la personal y, por último, la grupal. Estos resultados contrastan con los estudios de Sebastián y Sánchez (1999) y Hernández y Torres (2005) cuando expresan que las necesidades del alumnado de nuevo ingreso no sólo se relacionan con la información, sino también con la atención personalizada.

Es cierto que en los resultados obtenidos se aprecian algunas diferencias entre los estudiantes noveles y veteranos, pero, bajo nuestro punto de vista, no las suficientes, sobre todo cuando los alumnos de nuevo ingreso son los principales destinatarios de las acciones tutoriales en el centro.

Por otra parte, se precisa un cambio de cultura en la institución univesitaria que permita delimitar un marco organizativo-institucional en el que integrar la tutoría bajo la aceptación, el acuerdo, el consenso y el compromiso de todos los miembros de la comunidad universitaria. Investigaciones sobre evaluación de necesidades de orientación de los estudiantes universitarios en diferentes contextos en los últimos años, como las de Pérez, De Lara y Sebastián (1990), Lobato y Muñoz (1998), Arnaiz e Isus (1995), Castellano (1995), Sebastián y Sánchez (1999), Zamorano y Oliveros (1998) o Campoy y Pantoja (2000), resaltan la pertinencia de fomentar y poner en marcha iniciativas de orientación en el contexto universitario, concretándose en servicios de información académica, servicios de orientación, acciones de información y orientación para el acceso a la universidad y Planes de Acción Tutorial.

Para terminar, queremos señalar que la principal limitación de este trabajo es lo restringido que resulta al tratarse de una muestra representativa, pero de una única 
facultad, por lo que es difícil realizar generalizaciones. No obstante, a favor del mismo, sí podemos decir que esta iniciativa ha permitido sensibilizar a otras titulaciones para llevar a cabo un similar estudio y así comparar resultados. También puede considerarse un límite la muestra escogida, pero está expresamente seleccionada para contrastar alumnado que tiene contemplado en su plan de estudios la función tutorial (alumnos de nuevo ingreso que coinciden con alumnado de Grado) y aquellos que están finalizando sus estudios de planes antiguos y no tienen esta exigencia, pero sí se supone que más experiencia y se encuentran en otro momento de su desarrollo formativo.

Por todo ello, la prospectiva de este trabajo se debe centrar en ampliar el campo de actuación con otras universidades y comunidades autónomas, para poder extraer conclusiones generales en el ámbito de la Educación Superior. Igualmente, para el buen desarrollo de la acción tutorial, la implicación de las instituciones deberá centrarse en establecer el marco adecuado para el buen progreso de las mismas, además de empezar a pensar en establecer un tiempo y espacio en el horario de los profesores para esas funciones y diseñar un plan de formación para el profesorado, dado que progresivamente debe adaptarse a un nuevo rol para el que no está formado. Deberíamos considerar el EEES en el que estamos inmersos como una oportunidad de remover viejas estructuras y partir desde la formación para la recreación de una nueva universidad basada en el alumno como el centro del aprendizaje.

La tutoría es un derecho de los estudiantes, por lo que se convierte en una exigencia para la institución, pero, además, si se lleva a cabo de manera adecuada, es un elemento de calidad, de innovación y de futuro; por lo tanto, debemos continuar trabajando juntos para que su puesta en marcha contribuya realmente al desarrollo y formación integral del estudiante universitario.

\section{BIBLIOGRAFÍA}

Adame, M. T. (2002). La orientación y la tutoría en la universidad. Análisis de las realidades existentes. Educació i Cultura, 15, 215-232.

Alañón, M. T. (2000). La función tutorial. Madrid: ICE - Universidad Politécnica de Madrid.

Alcón, E. (2003). Tutoría personalizada y pedagogía reflexiva en el contexto universitario. En F. Michavila \& J. García (Eds.), La tutoría y los nuevos modos de aprendizaje en la universidad (pp. 85-93 ). Madrid: Cátedra UNESCO.

Almajano, M. P., \& Bertrán, E. (1999, diciembre). Formación de tutores de Universidad. Comunicación presentada en el I Symposium Iberoamericano de Didáctica Universitaria: la calidad de la docencia en la universidad. Santiago de Compostela, España.

Álvarez, P., Asensio, I., Forner, A., \& Sobrado, L. (2006). Los planes de acción tutorial en la universidad. En T. Escudero \& A. Correa (Coords.), Investigación e innovación educativa: algunos ámbitos relevantes (pp. 147-206 ). Madrid: La Muralla.

Álvarez, P., \& González, M. (2008). Análisis y valoración conceptual sobre los modelos de tutoría universitaria. Revista Interuniversitaria de Formación del Profesorado, 22(1), 48-70.

Álvarez, P., \& Jiménez, H. (2003). Tutoría universitaria. La Laguna: Universidad de La Laguna. 
Álvarez Pérez, P. (2002). La función tutorial en la universidad. Una apuesta por la mejora de la calidad de la enseñanza. Madrid: EOS.

Álvarez Pérez, P. (2005-2006). La tutoría y la orientación universitaria en la nueva coyuntura de la enseñanza superior: El Programa "Velero". Contextos Educativos, 8-9, 281-293.

Arbizu, F., Lobato, C., \& Castillo, L. (2005). Algunos modelos de abordaje de la tutoría universitaria. Revista de Psicodidáctica, 10(1), 7-21.

Arnaiz, P., \& Isus, S. (1995). La tutoría: organización y tareas. Barcelona: Graó.

Buendía, L., Colás, P., \& Hernández, F. (1998). Métodos de Investigación en Psicopedagogía. Madrid: McGraw Hill.

Cáceres P., \& Rodríguez, M. C. (2001, septiembre). Análisis y evaluación del programa de acogida en la universidad Politécnica de Valencia. Comunicación presentada en el X Congreso Nacional de Modelos de Investigación Educativa: Investigación y evaluación educativas en la Sociedad del Conocimiento. A Coruña, España.

Campoy, T. J., \& Pantoja, A. (2000). La Orientación en la Universidad de Jaén. Revista Española de Orientación y Psicopedagogía, 11(19), 77-106.

Cano, R. (2008). Modelo organizativo para la planificación y desarrollo de la tutoría universitaria en el marco del proceso de Convergencia Europea en Educación Superior. Revista Interuniversitaria de Formación del Profesorado, 22(1), 185-206.

Cano, R. (2009). Tutoría universitaria y aprendizaje por competencias: ¿Cómo lograrlo? Revista Interuniversitaria de Formación del Profesorado, 12(1), 181-204.

Castellano, F. (1995). La orientación educativa en la Universidad de Granada. Granada: Servicio de Publicaciones de la Universidad de Granada.

Cid, A., \& Pérez, A. (2006). La tutoría en la Universidad de Vigo según la opinión de los profesores. Revista de Investigación Educativa, 24(2), 395-421.

Coriat, M., \& Sanz, R. (2005). Orientación y tutoría en la Universidad de Granada. Granada: Servicio de Publicaciones de la Universidad de Granada.

Domínguez, F. J. (2004). Calidad de la enseñanza y espacio tutorial: La tutoría como instrumento dinamizador en el proceso de aprendizaje. Alicante: ICE - Vicerrectorado de Convergencia Europea y de Calidad (Universidad de Alicante).

Ferrer, J. (2003). La acción tutorial en la Universidad. En F. Michavila y J. García [Eds.], La Tutoría y los nuevos modos de aprendizaje en la Universidad (pp. 67-84). Madrid: Universidad Politécnica de Madrid - Cátedra UNESCO de gestión y política universitaria.

Filella, G. et al. (2001, septiembre). La acción tutorial en la Facultad de Ciencias de la Educación de la Universidad de Lleida. Comunicación Presentada en el X Congreso Nacional de Modelos de Investigación Educativa: Investigación y evaluación educativas en la Sociedad del Conocimiento. A Coruña, España.

Gairín, J., Feixas, M., Guillamón, C., \& Quinquer, D. (2004). La tutoría académica en el escenario europeo de la educación superior. Revista Interuniversitaria de Formación del Profesorado, 18(1), 61-77.

Gallego, S. (1997). Perfil del tutor universitario. En P. Apodaca, F. Arbizu, C. Lobato \& C. Olalde [Eds.], Comunicaciones del Congreso Orientación Universitaria y Evaluación de la Calidad (pp. 67-74). Lejona, País Vasco: Servicio Editorial Universidad del País Vasco. 
Gallego, S. (2006). La intervención tutorial en la Educación Superior. En S. Gallego y J. Riart (Coords.), La tutoría y orientación en el siglo XXI: Nuevas propuestas (pp. 185196). Barcelona: Octaedro.

García Nieto, N., Asensio, I. I., Carballo, R., García García, M., \& Guardia, S. (2004). Guía para la labor tutorial en la Universidad en el Espacio Europeo de Educación Superior. Madrid: MECD.

García Nieto, N., Asensio, I. I., Carballo, R., García García, M., \& Guardia, S. (2005). La tutoría universitaria ante el proceso de armonización europea. Revista de Educación, 337, 189-210.

González Maura, V., \& González Tirados, R. M. (2007). Competencias genéricas y formación profesional: un análisis desde la docencia universitaria. Revista Iberoamericana de Educación, 47, 185-209.

Hernández, M. E., \& Domínguez, D. (1998). La nueva acción tutorial en la enseñanza universitaria. Revista de Enseñanza Universitaria, 13, 79-88.

Hernández, V., \& Torres, J. (2005). La acción tutorial en la universidad. Recuperado de http://www.upcomillas.es/eees/Documentos/informe_acción_tutorial.pdf

Lapeña, C., Sauleda, N., \& Martínez, A. (2011). Los programas institucionales de acción tutorial: Una experiencia desarrollada en la Universiad de Alicante. Revista de Investigación Educativa, 29(2), 341-361.

Lázaro, A. (1997). La acción tutorial de la función docente universitaria. Revista Complutense de Educación, 8(1), 233-252.

Lázaro, A. (2002). La acción tutorial de la función docente universitaria. En V. Álvarez \& A. Lázaro (Coords.), Calidad de las universidades y orientación universitaria (pp. 249-282). Málaga: Aljibe.

Lázaro, A. (2003). Competencias tutoriales en la universidad. En F. Michavila \& J. García (Eds.), La tutoría y los nuevos modos de aprendizaje en la universidad (pp. 107128). Madrid: CAM - Cátedra UNESCO.

Ley 3/2005, de 25 de abril, de Universidades de la Región de Murcia. Boletín Oficial de la Región de Murcia, 11 de mayo de 2005, 106.

Ley Orgánica 1/1990, de 3 de octubre, de Ordenación General del Sistema Educativo. Boletín Oficial del Estado, 4 de octubre de 1990, 238.

Ley Orgánica 4/2007, de 12 de abril, por la que se modifica la Ley Orgánica 6/2001, de 21 de diciembre, de Universidades. Boletín Oficial del Estado, 13 de abril de 2007, 89.

Ley Orgánica 6/2001, de 21 de diciembre, de Universidades. Boletín Oficial del Estado, 24 de diciembre de 2001, 307.

Lobato, C. (2003). Estrategias y recursos para el desarrollo de la acción tutorial en la diversidad. En P. R. Álvarez y H. Jiménez (Comp.), Tutoría Universitaria (pp. 51-77). La Laguna, Santa Cruz de Tenerife: Universidad de la Laguna.

Lobato, C., Arbizu, F., \& Castillo, L. (2004). Representaciones de la tutoría universitaria en profesores y estudiantes: Estudio de un caso. Revista Educación XX1, 7, 135-168.

Lobato, C., \& Arbizu, F. (2003). Percepciones del profesorado y alumnado universitario respecto al proceso de enseñanza-aprendizaje. Campo abierto, 23, 69-84.

Lobato, C., \& Muñoz, M. (1998). Diagnóstico de necesidades de orientación universitaria. En E. Reppeto \& C. Velaz (Comp.), Orientación de la carrera, recursos humanos y mercado laboral. Actas del XV de la AIOEP (Vol. II, pp. 457-458). Madrid: UNED. 
Martínez, M., Buxarrais, M. R., \& Esteban, F. (2002). Ética y formación universitaria. Revista Iberoamericana de Educación, 29, 17-43.

Martínez, M., \& Crespo, E. (2009). La tutoría universitaria en el modelo de la convergencia europea. En C. Gómez \& S. Grau (Coords.), Propuestas de diseño, desarrollo e innovaciones currículares y metodológicas en el EEES (pp. 451-466). Alicante: Marfil.

Martínez Clares, P., Martínez Juárez, M., \& Pérez Cuso, J. (2012). El Espacio Europeo de Educación Superior y la tutoría universitaria. En M. A. Sánchez-Mora (Dir.), Tutoría Universitaria: Un estudio del Plan Especial de Tutorías en la Universidad de Murcia (pp. 75-90). Murcia: EDITUM.

Monreal, M. C. (1999, diciembre). La tutoría soporte de la educación: la tutoría en la Universidad. Comunicación presentada en el I Symposium Iberoamericano de Didáctica Universitaria: la calidad de la docencia en la Universidad. Santiago de Compostela, España.

Pérez, R., De Lara, E., \& Sebastián, A. (1990). Servicios de Orientación en la UNED: Demanda expresa y necesidades detectadas. Revista Iberoamericana de Educación, 3(1), 23-56.

Pérez Abellás, A. (2006). Percepciones de la tutoría en profesores y alumnos de la universidad. Síntesis de una investigación. Educación, Desarrollo y Diversidad, 9(2), 5-20.

Pino, M. R., \& Ricoy, M. C. (2006). La tutoría como recurso en la enseñanza universitaria. Estudio de necesidades y contribuciones actuales. Bordón, 58(3), 345-358.

Real Decreto 1791/2010, de 30 de diciembre, por el que se aprueba el Estatuto del Estudiante Universitario. Boletín Oficial del Estado, 31 de diciembre de 2010, 318.

Rodicio, M. L., Villayandre, A., \& Pérez, J. C. (2001). La tutoría en la Universidad de A Coruña: una aproximación a la utilización de este servicio por parte de los alumnos/ as. En X. Salvador \& M. L. Rodicio (Eds.), Actas del VII Simposio de Orientación (pp. 694-698). A Coruña: Servicio de Publicaciones de la Universidad de A Coruña.

Rodríguez Espinar, S. (2008). Manual de Tutoría Universitaria (2ª ed.). Barcelona: Octaedro/ ICE-UB.

Rodríguez Moreno, M. L. (2002). Hacia una nueva orientación universitaria: Modelos integrados de acción tutorial, orientación curricular y construcción del proyecto profesional. Barcelona: EUB.

Rumbo, B., \& Gómez, T. (2009, noviembre). La acción tutorial en la enseñanza universitaria. La experiencia de la Universidad de A Coruña. Comunicación presentada al II Congreso Internacional UNIVEST'09: Claves para la implicación de los estudiantes en la Universidad. Gerona, España.

Rumbo, B., \& Gómez, T. (2011). La acción tutorial en un contexto universitario masificado y la reivindicación europea de su valor formativo. Revista de Formación e Innovación Educativa Universitaria, 4(1), 13-34.

Salmerón, H., \& López, V. (2000). Orientación Educativa en las universidades. Granada: GEU.

Sanz Díaz, M.T. (2012). Estudio de la acción tutorial como paso previo a la implantación del EEES en la Licenciatura de Administración y Dirección de Empresas de la Universidad de Huelva. Revista de Investigación Educativa, 30(1), 145-160.

Sanz Oro, R. (2005). Integración del estudiante en el sistema universitario: La tutoría. Cuadernos de Integración Europea, 2, 65-95. 
Sanz Oro, R. (2009). Tutoría y atención personal al estudiante en la universidad. Madrid: Síntesis.

Sebastián, A., \& Sánchez, M. F. (1999). La función tutorial en la Universidad y la demanda de atención personalizada en la orientación. Educación XXI, 2, 245-263.

Sobrado, L. (2008). Plan de acción tutorial en los centros docentes universitarios: el rol del profesor tutor. Revista Interuniversitaria de Formación del Profesorado, 22(1), 89-108.

Villayandre, A., \& Pérez, J. (2000). Tutoría de iguales. En AA.VV, Congrés d'Orientació Universitària (pp. 233-241). Barcelona: EUB.

Zabalza, M. A. (2003). Competencias docentes del profesorado universitario: Calidad y desarrollo profesional. Madrid: Narcea.

Zabalza, M. A., \& Cid, A. (2006). La tutoría en la universidad desde el punto de vista del profesorado. Bordón, 58(2), 247-267.

Zamorano, R., \& Oliveros, L. (1998). Análisis de necesidades orientadoras en los alumnos de primer curso de la Facultad de Educación. En E. Reppeto \& C. Velaz (Comp.), Orientación de la carrera, recursos humanos y mercado laboral. Actas del XV de la AIOEP (Vol. II, pp. 487-498). Madrid: UNED.

Fecha de recepción: 6 de marzo de 2012.

Fecha de revisión: 6 de marzo de 2012.

Fecha de aceptación: 14 de enero de 2013. 